\title{
Once daily antibiotic regimen in paediatric oncology
}

\author{
E Bouffet, C Fuhrmann, D Frappaz, D Couillioud, V Artiges, C Charra, D Bouhour, \\ M Brunat Mentigny
}

\begin{abstract}
The feasibility and efficacy of a once daily antibiotic regimen were assessed in children with malignant tumours.

Over a 44 month period, 296 febrile episodes were treated with a regimen of once daily ceftriaxone-amikacin (and teicoplanin or vancomycin if the patient had a central line). The treatment was successful in $272(92 \%)$ episodes without modification of the antibiotic regimen, and only one patient died of uncontrolled sepsis. A once daily antibiotic regimen is therefore feasible and worthwhile in the treatment of febrile episodes in children with cancer.
\end{abstract}

(Arch Dis Child 1994; 70: 484-487)

The use of intensive treatment in paediatric oncology has led to major therapeutic advances, and most of the current protocols include multidrug chemotherapy with the risk of profound neutropenia. Infection is the major treatment related risk of intensive chemotherapy and has led to the development of several empirical antibiotic regimens. Many of the principles of the management of febrile neutropenic patients with cancer were developed during the early 1970s, centred around the mortality associated with Gram negative bacteria. ${ }^{1}$ The spectrum of infections in neutropenic patients is changing, however, with an increasing occurrence of Gram positive organisms, especially in patients with indwelling intravenous catheters. ${ }^{2}$ Although some trials in adult patients have indicated that monotherapy with ceftazidime is a viable alternative to a standard combination antibiotic regimen, ${ }^{3}$ the use of monotherapy versus a combination of two or three antibiotics remains controversial. Although numerous trials have evaluated a variety of schedules with single drugs or combination regimens, none has been shown to have a clear cut superiority. A broad spectrum of activity is the essential component of an empirical regimen, but toxicity, tolerance, and nursing time must also be considered. These objectives prompted the development of a simplified regimen based on a minimum number of daily injections.

\section{Patients and methods}

PATIENTS

Between July 1989 and February 1993, all children with solid tumours and lymphomas treated in the Centre Leon Bérard were eligible for the study. All febrile episodes, with or without neutropenia, were analysed. Fever was defined as a single axillary temperature higher than $38^{\circ} \mathrm{C}$, or a rectal temperature higher than $38.5^{\circ} \mathrm{C}$. All patients were examined on admission and at least once daily by a doctor during the treatment course. At enrolment samples of blood and urine were cultured and a chest radiograph was obtained. Blood cultures were performed daily as long as the patient remained febrile. A full blood count was performed initially for each patient, and then daily for neutropenic patients only (neutrophil count less than $0.5 \times 10^{9} / 1$ polymorphonuclear cells and band forms). Serum levels of creatinine, urea, electrolytes, and proteins were measured on the day of admission, and once a week during the study.

\section{ANTIBIOTIC TREATMENT}

The antibiotic regimen combined ceftriaxone, amikacin, and teicoplanin or vancomycin if the patient had a central line. The protocol was approved by the Centre Leon Bérard drug committee.

Ceftriaxone was administered at a dose of 60 $\mathrm{mg} / \mathrm{kg}$ body weight (with a maximum daily dose of $2 \mathrm{~g}$ ) in a single daily dose as a bolus injection. Amikacin was administered at a dose of $15 \mathrm{mg} / \mathrm{kg}$ body weight in a single daily dose. The amikacin concentrations were measured one hour after the end of the 30 minute infusion, and just before the next daily dose. Serum concentrations were determined by fluorescence polarisation immunoassay (TDX, Abbot Laboratories). Teicoplanin was administered at a loading dose of $12 \mathrm{mg} / \mathrm{kg}$ body weight every 12 hours for 24 hours, and then $6 \mathrm{mg} / \mathrm{kg}$ body weight in a single daily dose. For financial reasons teicoplanin was replaced by vancomycin $(20 \mathrm{mg} / \mathrm{kg}$ body weight twice a day) from February 1990.

Amphotericin B was added if a patient with neutropenia had persistent fever after 72 hours of empirical antibiotic treatment, or if there was clinical or microbiological evidence of invasive fungal infection. Acyclovir was added to the antimicrobial regimen if there was clinical evidence of viral infection or a severe mucositis.

The antibiotic regimen was prolonged until the recovery of the neutrophil count in neutropenic patients. For patients without neutropenia the treatment was interrupted after 48 hours if there was no bacteriological confirmation. Microbiologically documented infections were treated for 10 
days after negative blood cultures had been obtained.

Episodes of fever were classified as due to (a) microbiologically documented infection, with or without bacteraemia, (b) clinically documented infection, or (c) fever of unknown origin according to the definition used by the EORTC group. ${ }^{4}$

Response to the antibiotic regimen was classified as (a) success when the patient improved, fever and signs of infection resolved, and the infective micro-organism was eradicated without a change in the antibiotic regimen, or (b) treatment failure if the patient died during treatment or had no clinical or microbiological response to the empirical treatment, requiring a change in antibiotic treatment.

\section{Results}

Over a 44 month period, 155 patients (mean age 8.7 years, range 3 months to 20 years) were entered into the analysis for a total of 303 febrile episodes. The initial disease was lymphoma (35 patients), neuroblastoma (25 patients), brain tumour (21 patients), osteosarcoma (20 patients), rhabdomyosarcoma (15 patients), Wilms' tumour (11 patients), Ewing's sarcoma (seven patients), and other tumours (21 patients). Seven episodes were excluded from the analysis for violation of the protocol: five received antibiotics three times a day, and two presented a life threatening infection with multiple organ failure requiring transfer to an intensive care unit. The remaining 296 episodes of fever included 236 neutropenic episodes. In 232 episodes the patient had a central line and was initially treated with a regimen containing a glycopeptide. One hundred and ninety one patients received vancomycin and 43 patients teicoplanin. The average duration of neutropenia was 6.7 days (range 1-37 days). The average duration of fever was 3.2 days (range 1-19 days) and the average duration of antibiotic treatment was eight days (range 2-28 days).

Ninety six (32\%) episodes were microbiologically documented. Of these 96 episodes, $82(85 \%)$ were documented infections with bacteraemia. Three episodes were due to multiple organisms and two to candida fungaemia. Gram positive cocci accounted for $59(77 \%)$ of the episodes of single organism bacteraemia and Gram positive rod forms for $20(26 \%)$. Twenty six (9\%) episodes were clinically documented infection without bacteraemia. The remaining 174 (59\%) episodes were classified as fever of unknown origin. The table shows the characteristics of the 96 microbiologically documented infections.

Empirical antibiotic treatment was unchanged in $272(92 \%)$ episodes. Persistent fever or microbiologically documented fungal infection, or both, led to additional amphotericin B treatment in $44(15 \%)$ episodes. Acyclovir was added in $62(21 \%)$ episodes, mostly for severe mucositis. The treatment failed in 24 episodes. The treatment failures were due to either primary resistance to ceftriaxone (six episodes) or persistence of the bacteraemia (five episodes), 10 episodes clinical evidence of inefficacy, two with development of a secondary infection, and one with vancomycin intolerance. Ten patients switched from ceftriaxone to ceftazidime and three from ceftriaxone to imipenem. The treatment failed in 13 (14\%) of 96 microbiologically documented episodes, in six (23\%) of 26 clinically documented episodes, and in only five (3\%) of 174 episodes of fever of unknown origin. Treatment of Gram negative sepsis had a significantly higher failure rate than treatment of Gram positive sepsis (36\% v 8\%; $\mathrm{p}<0.001)$. Thirty one $(20 \%)$ patients received their treatment in a once daily ambulatory schedule, requiring a short daily admission to hospital. Eleven $(7 \%)$ patients with contamination of their central line with a Staphylococcus epidermidis infection had catheters removed to shorten the duration of the admission to hospital. Two hundred and ninety five episodes resolved, including 23 in which the initial treatment had failed and the patient had been switched to another combination of drugs. One patient died of a multiresistant Pseudomonas maltophila related sepsis.

\section{AMIKACIN CONCENTRATIONS}

Data were available for pharmacokinetic evaluation in $34(11 \%)$ episodes. The mean (SD) peak concentration of amikacin was 23.9 $(10 \cdot 1) \mathrm{mg} / \mathrm{l}$, whereas the corresponding value for the trough was $0.6 \mathrm{mg} /$. Repeated doses in 25 patients did not show any significant changes during treatment for the peak nor trough concentrations. Pharmacokinetic results in 27 patients with and seven without neutropenia were not significantly different: 22 and $27 \mathrm{mg} / 1$ respectively for peak concentrations, and $0 \cdot 6$ $\mathrm{mg} / \mathrm{l}$ in each group for the trough concentrations. The serum concentrations of amikacin varied greatly between patients, ranging from 6.7 to $52.6 \mathrm{mg} / \mathrm{l}$ for the peak concentration. Fifty five per cent of the patients had a peak concentration less than $25 \mathrm{mg} / 1$, and only $10 \%$ had a peak concentration greater than $40 \mathrm{mg} / \mathrm{l}$. Trough concentrations were less than the measurable limits in $85 \%$ of the patients.

Characteristics of the 96 microbiologically documented infections (including three episodes with multiple organisms)

\begin{tabular}{ll}
\hline Organism & $\begin{array}{l}\text { No of } \\
\text { positive cultures }\end{array}$ \\
\hline Bacteraemia & \\
Staphylococcus epidermidis & 40 \\
Staphylococcus aureus & 6 \\
Other staphylococci & 6 \\
Streptococci group D & 8 \\
Streptococci non-group D & 3 \\
Pseudomonas & 6 \\
Escherichia coli & 4 \\
Klebsiella & 3 \\
Other Gram negative & 6 \\
Anaerobes & 3 \\
Fungaemia & 2 \\
Candida & 12 \\
Urinary tract infection & 99 (96 episodes) \\
Total &
\end{tabular}


TOXICITY

Despite the addition of other potentially nephrotoxic drugs (for example, amphothericin B), nephrotoxicity was minimal. The median serum creatinine concentration before and after treatment was 36 (range 12-109) and 34 (range 15-96) $\mu \mathrm{mol} / \mathrm{l}$. Auditory function was not evaluated, although no clinical alteration was detected during or after amikacin treatment in any patient.

\section{Discussion}

The benefit of antibiotic treatment has been well established in patients with cancer receiving intensive chemotherapy. ${ }^{1}$ During the first few days after a febrile episode, the initial regimen is truly empirical. Most empirical antibiotic regimens include an aminoglycoside (for example, gentamicin, tobramicin, or amikacin), usually in combination with a penicillin against Gram negative bacteria, and often with a glycopeptide (for example, vancomycin or teicoplanin) against staphylococcal bacteria. $^{15}$ As most studies have evaluated patients with respect to the success of treatment (alive versus dead) at 48 or 72 hours after entry, no regimen has shown a clear cut superiority. Many recent investigations have focused on the potential use of simplified schedules. Pizzo et al, comparing an initial single drug treatment with ceftazidime with a standard combination antibiotic treatment, showed that the former was successful as an empirical treatment of fever in most patients with neutropenia, although a significant number required the subsequent addition of vancomycin. ${ }^{3}$ In contrast, Kramer et al ${ }^{6}$ and the EORTC group ${ }^{4}$ concluded that ceftazidime alone was inadequate in their patients with cancer, and suggested the need to combine either a drug against Gram positive bacteria (Kramer et $a l^{6}$ ) or an aminoglycoside $\left(\right.$ EORTC study ${ }^{4}$ ) to prevent failures and the development of secondary infection. In our experience, the high incidence of Gram positive infection among patients with central lines led us to include a glycopeptide in our initial empirical regimen. To simplify the schedule, we choose three drugs which can be administered only once every 24 hours. Ceftriaxone and teicoplanin have long half lives, allowing once daily administration. ${ }^{78}$ Studies have suggested once daily dosing with an aminoglycoside to be at least as effective and as safe as the conventional dosing regimen. ${ }^{910}$ This regimen had the obvious advantage of greater convenience, allowing outpatient treatment, and, as a consequence, economic advantages. ${ }^{11}$ The higher cost of teicoplanin compared with vancomycin, however, led us to choose the latter after six months of experience, and to reserve teicoplanin for outpatients only. Our data show a high incidence of Gram positive bacteria ( $72 \%$ of positive blood isolates) and a particularly high number of $S$ epidermidis (47\% of isolates). The wide use of indwelling central venous catheters and the low ratio of nurses per bed (eight day nurses for a 20 bed unit) contributes to this pattern of infection. ${ }^{12}$ Ceftriaxone, a third generation cephalosporin, is not considered to act against pseudomonas bacteria. ${ }^{13}$ This necessitates the aminoglycoside association and resulted in frequent changes to more specific drugs among patients with Gram negative infections (eight of 22 cases). Most of the changes occurred within 48 hours after the institution of the empirical antibiotic treatment, and one patient died only a few hours after the beginning of antibiotic treatment because of a multiple organ failure due to $P$ maltophila.

As a significant percentage of febrile neutropenic patients who are treated with an empirical antibiotic have neither a specific infection nor respond immediately to the treatment, an appropriate question is whether or not these patients require a modification of their treatment. Several efforts have focused on the identification of high and low risk patients with unexplained fever. ${ }^{214}$ Most of the patients in our study had a short duration of neutropenia (less than seven days), and only five $(3 \%)$ of 174 episodes of unexplained fever required a modification of their treatment based on clinical features (for example, persistent chills and rigors). As no formal recommendation for antibiotic modification was proposed for persistent well tolerated fever, most of the patients completed successful treatment without modification of the regimen. Fifteen per cent, however, required an additional antifungal drug, and $21 \%$ the addition of acyclovir. Only two patients developed a secondary infection (due to pseudomonas and candida respectively) while receiving ceftriaxone, amikacin, and vancomycin.

Amikacin has been shown to be effective at a dose of $7.5 \mathrm{mg} / \mathrm{kg}$ every 12 hours. Studies suggest that single high daily doses of aminoglycoside are less nephrotoxic, maximising the therapeutic effects of these dose dependent drugs. ${ }^{15-17} \mathrm{New}$ recommendations for peak and trough concentrations of amikacin need to be formulated for this once daily regimen, however. These should be based on pharmacokinetic, pharmacodynamic, efficacy, and toxicity data. Our study shows a large variability in children, probably due to the wide age range of patients included ( 3 months to 20 years). The pharmacokinetic parameter obtained in our study showed lower concentrations in children with febrile neutropenia. An increased aminoglycoside dose requirement has been reported in patients with haematological malignancies with neutropenic fever. ${ }^{18} 19$ Dose recommendations for patients with febrile neutropenia are $7 \cdot 5-10 \mathrm{mg} / \mathrm{kg}$ every eight hours in these reports. Viscoli et al, in a study of neutropenic children undergoing bone marrow transplantation, administered amikacin at a single daily dose of $20 \mathrm{mg} / \mathrm{kg}^{20}$ The peak concentration measured 30 minutes after the end of infusion was $36.9(9.5) \mathrm{mg} / \mathrm{l}$ and the trough concentrations were less than the measurable limit at six hours in 13 of 16 courses. In children without cancer with Gram negative infections, Kafetzis et al reported a peak concentration (measured 
one hour after the end of infusion) of 36.5 (9) $\mathrm{mg} / \mathrm{l}$ and a concentration at 12 hours below the measurable limit in all patients after a single daily dose of $20 \mathrm{mg} / \mathrm{kg}$. ${ }^{21}$ Although the recommended dose might be $20 \mathrm{mg} / \mathrm{kg}$ in children treated with once daily amikacin, more detailed studies of pharmacokinetics in children must define more precisely the optimum peak concentration that provides the greatest effectiveness with the least toxicity. Data on toxicity are limited. Nephrotoxicity was negligible, and there was no clinical auditory impairment in any patient.

In conclusion, our study suggests that a single daily dose of ceftriaxone and amikacin with or without a glycopeptide is feasible in children being treated for solid tumours and lymphomas. This treatment is, however, not recommended for life threatening infections or documented Gram negative septicaemia, or both. These results are encouraging and suggest that, for a selected population, antibiotic treatment can be administered as a simple outpatient schedule. There is still a need to identify the optimum aminoglycoside dose, and whether such a single daily empirical treatment is appropriate in prolonged neutropenia for patients treated for haematological malignancies, or undergoing bone marrow transplantation.

1 Rubin RH. Empiric antibacterial therapy in granulocytopenia induced by cancer chemotherapy. Ann Intern Med 1988; 108: 134-6.

2 Rubin M, Hathorn JW, Pizzo PA. Controversies in the management of febrile neutropenic cancer patients. Cancer Invest 1988; 6: 167-84

3 Pizzo PA, Hathorn JW, Hiemenz J, et al. A randomized trial comparing ceftazidime alone with combination antibiotic therapy in cancer patients with fever and neutropenia. therapy in cancer patients with

4 The EORTC International Antimicrobial Therapy Cooperative Group. Ceftazidime combined with a short or long course of amikacin for empirical therapy of gramnegative bacteremia in cancer patients with granulocytopenia. N Engl F Med 1987; 317: 1692-8.

5 Shenep JL, Hughes WT, Roberson PK, et al. Vancomycin, ticarcillin, and amikacin compared with ticarcillinclavulanate and amikacin in the empirical treatment of febrile, neutropenic children with cancer. $N$ Engl $f$ Med 1988; 319: 1053-8.

6 Kramer BS, Ramphal R, Rand KH. Randomized comparison between two ceftazidime-containing regimens and cephalothin-gentamicin-carbenicillin in febrile granulocephalothin-gentamicin-carbenicillin in febrile granulocytopenic cance

7 Patel IH, Kaplan SA. Pharmacokinetic profile of ceftriaxone in man. Am f Med 1984; 77: 17-25.

8 Traina GL, Bonati M. Pharmacokinetics of teicoplanin in man after intravenous administration. If Pharmacokinet Biopharm 1984; 12: 119-28.

9 Trujillo H, Robledo J, Robledo C, et al. Single daily dose amikacin in paediatric patients with severe gram-negative infections. F Antimicrob Chemother 1991; 27 (suppl C): 141-7.

10 Meunier F, Van der Auwera P, Aoun M, Ibrahim S, Tulkens PM. Empirical antimicrobial therapy with a single daily dose of ceftriaxone plus amikacin in febrile single daily dose of ceftriaxone plus amikacin in febrile
granulocytopenic patients: a pilot study. $\mathcal{F}$ Antimicrob granulocytopenic patients: a pilot study
Chemother 1991; 27 (suppl C): 129-39.

11 Martino P, Girmenia C, Raccah R, Micozzi A, Cimino G, Mandelli F. Ceftriaxone and amikacin as single daily dose in the empiric therapy for febrile episodes in neutropenic patients. Haematologica 1990; 75: 69-74.

12 Rubin M, Hathorn JW, Marshall D, Gress J, Steinberg SM, Pizzo PA. Gram-positive infections and the use of vancomycin in 550 episodes of fever and neutropenia. Ann Intern Med 1988; 108: 30-5.

13 Barriere SL, Flaherty JF. Third-generation cephalosporins: a critical evaluation. Clin Pharm 1984; 3: 351-73.

14 Pizzo PA. After empiric therapy: what to do until the granulocyte comes back. Rev Infect Dis 1987; 9: $214-9$.

5 Tulkens PM. Pharmacokinetic and toxicological evaluation of a once-daily regimen versus conventional schedules of netilmicin and amikacin. F Antimicrob Chemother 1991; 27 (suppl C): 49-61.

16 Van der Auwera P. Pharmacokinetic evaluation of single daily dose amikacin. F Antimicrob Chemother 1991; 27 (suppl C): 63-71.

17 Powell SH, Thompson WL, Luthe MA, et al. Once-daily vs. continuous aminoglycoside dosing: efficacy and toxicity in animal and clinical studies of gentamicin, netilmicin, and animal and clinical studies of gentamicin, netil

18 Zeitany RG, El Saghir N, Santhosh KC, Sigmon MA. Increased aminoglycoside dosage requirements in hematologic malignancy. Antimicrob Agents Chemother 1990; 34: 702-8.

19 Kaojarern S, Maoleekoonpairoj S, Atichartakarn V. Pharmacokinetics of amikacin in hematologic malignancies. Antimicrob Agents Chemother 1989; 33: 1406-8.

20 Viscoli C, Dudley M, Ferrea G, et al. Serum concentrations and safety of single daily dosing of amikacin in children and safety of single daily dosing of amikacin in children Chemother 1991;27 (suppl C): 113-20.

21 Kafetzis DA, Sianidou L, Vlachos E, et al. Clinical and pharmacokinetic study of a single daily dose of amikacin in paediatric patients with severe gram-negative infections. F Antimicrob Chemother 1991; 27 (suppl C): 105-12. 